\title{
Influence of spent ginger yeast cultures on the production performance, egg quality, serum composition, and intestinal microbiota of laying hens
}

\author{
Junhan Liu ${ }^{1}$, Yuhong Jin $^{1, *}$, and Junhua Yang ${ }^{2}$
}

\footnotetext{
* Corresponding Author: Yuhong Jin Tel: +86-15163803635,

E-mail: yuhongjin79@sdau.edu.cn

${ }^{1}$ Key Laboratory of Food Processing Technology and Quality Control in Shandong Province, College of Food Science and Engineering, Shandong Agricultural University, Tai'an 271018, China

${ }^{2}$ Institute for Agri-Food Standards and Testing Technology, Shanghai Academy of Agricultural Sciences, Shanghai 201100, China
}

ORCID

Junhan Liu

https://orcid.org/0000-0002-8880-5289

Yuhong Jin

https://orcid.org/0000-0002-4387-7019

Junhua Yang

https://orcid.org/0000-0002-1554-0275

Submitted Nov 19, 2021; Revised Dec 30, 2021; Accepted Feb 1, 2022
Objective: Spent ginger is a byproduct of juice extraction from the rhizome of ginger (Zingiber officinale). Despite its nutritional value, it is difficult to preserve or further process and thus is often wasted. This study uses spent ginger as a substrate for fermentation and cultivates spent ginger yeast cultures (SGYCs) that are then added to the feed of laying hens. The effects of SGYCs on production performance, egg quality, serum composition, and intestinal microbiota of laying hens were investigated.

Methods: Eighty 60-week-old Hy-Line Brown hens were separated into 5 experimental groups with 4 replicates per group ( 4 hens per cage, 4 cages per replicate). The control group was fed a basal diet while experimental groups were also given SGYCs at the levels of $5,10,20$, and $40 \mathrm{~g} / \mathrm{kg}$ for 6 weeks.

Results: The addition of SGYCs significantly increased the laying rate and nutrient digestibility, decreased feed conversion ratio, and enhanced the color of egg yolks $(p<0.05)$. No changes were observed in activity levels of alanine aminotransferase and aspartate aminotransferase in the serum ( $>0.05$ ), but the activities of superoxide dismutase, glutathione peroxidase, and peroxidase all significantly increased, and contents of malondialdehyde were significantly reduced $(p<0.05)$. In addition, changes in the relative abundance of Firmicutes and Bacteroidetes might be the main factor contributing to the significant increase in the apparent digestibility of crude protein and crude fat in laying hens $(\mathrm{p}<0.05)$. Conclusion: The current evidence shows that dietary supplementation of SGYCs to the feed of laying hens can improve laying rates, enhance antioxidative defenses, and influence dominant intestinal bacteria.

Keywords: Blood Biochemical; Egg Quality; Intestinal Microbiota; Laying Hens; Laying Rate; Spent Ginger Yeast Cultures

\section{INTRODUCTION}

Eggs are considered an ideal food for humans in terms of nutritional value. Egg protein has amino acid composition highly suitable and can be easily absorbed by the human body, with utilization rates up to $98 \%$ [1]. Eggs are also rich in lecithin, sterols, and various micronutrients [2]. The egg industry is of significant economic importance, with over 70 million tons produced annually across the world [3]. Increasing the output and quality of egg production remains of high interest in agricultural research.

Supplementation to the diet of laying hens is a common strategy to improve egg production. Yeast cultures (YCs), which typically refer to mixtures of both yeast (Saccharomyces cerevisiae [S. cerevisiae]) and the solid or liquid mediums in which they were fermented, are considered high-quality supplements because they contain various bioactive substances including yeast cells, vitamins, amino acids, organic acids, and oligosaccharides [4]. Re- 
cent research has shown that the addition of YCs in poultry feeds often has positive effects. Gómez et al [5] demonstrated that YCs supplements improved feed conversion ratio, breast yield, and ileal digestibility of dry matter in broiler chicks. Ma et al [6] have shown that the addition of distiller's grain based YCs to the diet of broiler chicks results in rapid increases in body weight, and Yalçin et al [7] showed that inclusion of commercial YCs in the diet of laying hens can decrease cholesterol levels in the yolk while also increasing the overall weight of the eggs.

Spent ginger is a byproduct of commercial processes that crush the rhizome of ginger (Zingiber officinale) to extract juices. Due to the high moisture content in spent ginger, it is difficult to preserve or further process, and thus often discarded. However, it is also an ideal substrate to culture YCs for feed supplementation. Large molecules such as proteins and starches in spent ginger can be broken down into by yeast, which is conducive to absorption. As well, ginger contains various monoterpenes (cineole, citral, limonene, and $\alpha / \beta$-pinenes), sesquiterpenes ( $\beta$-elemene, farnesene and zerumbone), phenols (gingerols, [6]-shogaol, [6]-paradol and zingerone), and diphenylheptane compounds [8], known to have various health benefits including anti-inflammatory [9], anti-oxidization [10], anti-bacterial [11], blood sugar reducing [12], and gastrointestinal protecting effects [13]. Yeast cultures fermented in spent ginger are therefore expected to have various nutritional and medicinal benefits, making them a valuable feed additive.

Currently, research regarding the cultivation of YCs using spent ginger as a substrate, or the application of such cultures as a feed additive, is lacking. The present study aims to evaluate how the addition of spent ginger yeast cultures (SGYCs) to the diet of laying hens may affect their blood indicators and intestinal bacteria, as well as their productivity and the quality of eggs produced. We also explore the potential mechanisms through which SGYCs influence the output and quality of egg production. Our work aims to reduce the waste of ginger byproducts and promote the sustainable development of animal husbandry.

\section{MATERIALS AND METHODS}

\section{Animal care}

The present experiment was reviewed and approved by the Institutional Animal Care and Use Committee at Shanghai Academy of Agricultural Sciences (SAASPZ0920001).

\section{Materials}

Hy-Line Brown laying hens (60 weeks of age) were provided by Jun'an Egg Farm (Shanghai, China). Spent ginger yeast cultures were provided by the Fermentation Engineering Laboratory of Shandong Agricultural University (Taian,
China). Cultivation procedures were as follows: spent ginger was mixed with water at a 1:3 ratio, to which sucrose was added at $102 \mathrm{~g} / \mathrm{L}$ and S. cerevisiae (WET 137; Eaton, Nettersheim, Germany) at $0.4 \mathrm{~g} / \mathrm{L}$. The mixture was fermented in a semi-solid state at $20^{\circ} \mathrm{C}$ until sugar contents were less than 4 $\mathrm{g} / \mathrm{L}$ and no longer changed for two consecutive days, then squeezed with a plate and frame press. The pulp was dried at $55^{\circ} \mathrm{C}$ and crushed. The proximate composition of SGYCs was as follows: $9.6 \%$ moisture, $9.8 \%$ crude protein, $5.2 \%$ crude fat, $24.5 \%$ crude fiber, $6.0 \%$ ash, and $45.0 \%$ total crude carbohydrates.

\section{Animals, diets, and experimental design}

A total of $80 \mathrm{Hy}$-Line Brown laying hens were randomly allocated to one of five treatment groups. Each treatment had 4 replicates with 4 hens per replicate. The basal diet of the five treatment groups were supplemented with SGYCs at levels of 0 (control), 5, 10, 20, and $40 \mathrm{~g} / \mathrm{kg}$. All experimental diets were approximately isonitrogenous and isocaloric and were formulated to meet or exceed nutrient requirements recommended by the NRC [14]. Ingredients and nutrient composition of the basal diet are shown in Table 1.

The study was conducted for 8 weeks, including 2 weeks of adaptation and 6 weeks of the experiments. The laying

Table 1. Ingredients and nutrient composition of basal diet ( $\mathrm{g} / \mathrm{kg}$ as fed unless noted)

\begin{tabular}{lc}
\hline Item & Content \\
\hline Ingredients & \\
Corn & 600.0 \\
Soybean meal & 210.0 \\
Soybean oil & 15.0 \\
CaHPO $_{4}$ & 10.0 \\
Limestone & 80.0 \\
Fish meal & 20.6 \\
Wheat bran & 50.0 \\
NaCl & 3.2 \\
DL-Methionine & 1.2 \\
Premix & 10.0 \\
Nutrient composition & \\
Crude protein & 161 \\
Crude fat & 25 \\
Crude fiber & 40 \\
Ash & 120 \\
Calcium & 33.5 \\
Total phosphorus & 7.0 \\
Lysine & 9.8 \\
Methionine+cysteine & 8.0 \\
ME (Kcal/kg) & 2,850 \\
\hline
\end{tabular}

$\mathrm{ME}$, metabilizable energy.

1) Supplied per kilogram of diet: vitamin $A, 12,000 \mathrm{IU}$; vitamin $\mathrm{D}_{3}, 4,000 \mathrm{IU}$; vitamin $\mathrm{E}, 30 \mathrm{IU}$; vitamin $\mathrm{K}_{3}, 4 \mathrm{mg}$; vitamin $\mathrm{B}_{6}, 5 \mathrm{mg}$; vitamin $\mathrm{B}_{12}, 0.1 \mathrm{mg}$; thiamine, $2 \mathrm{mg}$; riboflavin, $8 \mathrm{mg}$; niacin, $45 \mathrm{mg}$; biotin, $0.2 \mathrm{mg}$; pantothenic acid, $11 \mathrm{mg}$; folic acid, $2 \mathrm{mg}$; Mn, 80 mg; Fe, 100 mg; Zn, 80 mg; Cu, 8 mg; l, 0.8 mg; Se, 0.3 mg. 
hens were housed in an environmentally controlled room where the temperature was $20^{\circ} \mathrm{C} \pm 2^{\circ} \mathrm{C}$. A 16 -h lighting schedule (16 light:8 dark) was used during the entire experiment. Hens were raised in cages: each cage was $400 \mathrm{~mm}$ long, 450 $\mathrm{mm}$ deep, $450 \mathrm{~mm}$ high in the front and $380 \mathrm{~mm}$ high in the rear. The bottom of the cage leaned 7 degrees and was equipped with a nipple drinker and a trough feeder. Each cage had 4 laying hens, which could eat and drink freely. Feeding, egg collection, and cleaning of the coop were performed daily. Feces were cleaned and the chicken coop was sprayed and disinfected once a week.

\section{Laying performance and egg quality traits}

During the feeding trial, the total number of eggs and total egg weight of each replicate were recorded every day. Feed consumption of each replicate was recorded each week. The average egg weight ( $\mathrm{g}$ ), laying rate (\%), average daily feed intake $(\mathrm{g})$, and feed conversion ratio of each replicate were calculated at 3-week intervals. The average egg weight was the ratio of the total number of eggs to the total egg weight. The laying rate refers to the number of hens that laid eggs as a percentage of the total number of laying hens. The feed conversion ratio was calculated by dividing the weight of feed consumed by the weight of eggs produced.

To determine egg quality, 12 eggs were randomly selected from each group on day 21 and day 42 of the feeding experiment. The major and minor axes of the eggs were measured using the egg shape index meter (FHK, Tokyo, Japan), and the egg shape index (long diameter/short diameter of eggs) was calculated. An egg quality tester (DET-6000; Nabel, Kyoto, Japan) was used to determine eggshell hardness (kgf), yolk color, albumen height (mm) and the Haugh unit. The determination of yolk color value was based on the "Roche color fan (RCF)" system, which consists of 15 color samples corresponding to values 1 to 15 . The thickness of the shell at the blunt end, tip and middle of the egg was measured using an eggshell thickness tester (C1012XBS; Mitutoyo corp, Kanagawa, Japan) and used to calculate average eggshell thickness.

\section{Apparent digestibility of nutrients}

Apparent digestibility was determined using the total feces collection method [15]. After the end of the feeding experiment period, 8 laying hens were randomly selected from each group to be unfed for 2 days to empty the original feces in the intestines, then re-fed for 2 days and had their feces collected for 4 consecutive days after feeding. Feathers and other foreign objects were removed from the collected feces, after which the feces were dried to a constant weight in an oven at $60^{\circ} \mathrm{C}$ and then pushed through a sieve $(0.425 \mathrm{~mm})$ using a sealed pulverizer (XL-20B; Xulang Machinery, Guangzhou, China). Crude fat (No. 920.39), crude protein (No.
978.04), crude fiber (No. 978.10), and ash (No. 930.05) were determined following methods of the Official Society of Analytical Chemists [16]. Apparent nutrient digestibility was calculated as follows:

$$
\begin{aligned}
& \text { Nutrient digestibility } \\
& =[(\text { nutrient intake }- \text { nutrient voided }) \\
& \quad /(\text { nutrient intake })] \times 100 \%
\end{aligned}
$$

Nutrient intake $=$ nutrient in $\operatorname{diet} \times$ feed intake

Nutrient voided

$=$ nutrient in feces $\times$ amount of feces voided

\section{Routine blood indices and serum biochemical parameters}

On the 42nd day of the experimental period, 12 laying hens were randomly selected from each treatment group of 16 . Blood samples were collected from the wing vein of each hen using disposable needles, and then placed in a vacuum sealed sampling vessel. A blood cell analyzer (MEK-6400C; Celltaca, Tokyo, Japan) was used to determine routine indices in the whole blood, including red blood cells (RBC), hemoglobin (HGB), hematocrit (HCT), mean corpuscular volume (MCV) and platelet count (PLT). Blood biochemical indicators were determined using the serum. Blood samples were centrifuged $(15 \mathrm{~min}, 3,000 \mathrm{rpm})$ at $4^{\circ} \mathrm{C}$ in a centrifuge (AG 22331; Hamburg, Eppendorf, Germany) to obtain serum samples, which were stored at $-80^{\circ} \mathrm{C}$. The following biochemical indicators were determined using an automatic biochemical analyzer (AU5800; Beckman Coulter, Brea, CA, USA): glucose, alanine aminotransferase (ALT), aspartate aminotransferase (AST), total protein (TP), and total cholesterol (CHOL).

\section{Serum antioxidant capacity}

The serum samples prepared in the previous section were used to determine the serum antioxidant capacity. The contents of glutathione peroxidase (GSH-Px), superoxide dismutase (SOD), peroxidase (CAT), and malondialdehyde (MDA) in the serum were determined using a microplate reader (Multiskan Sky; Thermo Fisher, Waltham, MA, USA) and a Beyotime Biotechnology testing kit (Beyotime Biotechnology, Shanghai, China), following manufacturer's instructions.

\section{Analysis of intestinal microbiota of laying hens}

On the 42nd day of the experimental period, 4 laying hens were randomly selected from each group to collect fresh feces. The fresh feces were placed in a sterile cryotube and placed in liquid nitrogen for rapid freezing, then sent to the laboratory to be stored in at $-80^{\circ} \mathrm{C}$ for subsequent analyses. 
Fecal samples were separately iced and homogenized, and each was processed using a QIAamp DNA Stool Mini Kit (Qiagen, Hilden, Germany) in strict accordance with the manufacturer's standard procedures to extract microbial genomic DNA. Primers 338F (5'-ACTCCTACGGGAGCAG CA-3') and 806R (5'-GGACTACHVGGGTWTCTAAT -3') were used for polymerase chain reaction amplification to detect V3-V4 regions of bacterial 16S rRNA. Sequencing and bioinformatics analysis were performed by PersonalBio Ltd. (Shanghai, China). Briefly, paired-end sequencing of community DNA fragments was conducted on the Illumina Miseq platform, and the original sequencing data was saved in FASTQ format. Sequences were quality filtered, denoised, merged, and had chimeras removed using the DADA2 plugin [17]. Sequences from all samples were sorted by abundance, after which they were used to select operational taxonomic units (OTUs) at a threshold of $97 \%$ similarity. Finally, the output of the representative sequences and OTUs were organized into a table. The Greengenes Database (Release 13.8) was used to classify the representative sequences of each OTU. Based on results from taxonomic classification of the OTU representative sequences, the composition table of the microbiota community in each sample at each classification level was summarized and visualized with a stacked bar chart.

\section{Statistical analysis}

Statistical analyses were performed using SAS 9.2. A oneway analysis of variance was performed to detect significance differences among groups. Means were also compared using Tukey's multiple comparison test to determine pair-wise differences. The linear and quadratic responses were analyzed using orthogonal polynomials. Differences at $\mathrm{p}<0.05$ were considered significant, whereas p-values between 0.05 and 0.1 were interpreted as trends. The Spearman rank correlation coefficient calculated in IBM SPSS Statistics 25 was used to analyze correlations, with $\mathrm{p}<0.05$ indicating significant correlation and $\mathrm{p}<0.01$ indicating extremely significant correlation.

\section{RESULTS AND DISCUSSION}

\section{Laying performance and egg quality traits}

As seen in Table 2, while SGYC supplementation had no significant effects on average egg weight or average daily feed intake ( $p>0.05$ ), it had a significant effect on the laying rate and feed conversion ratio. In the latter half of the experiment, the relationship between laying rate and SGYC supplementation followed a quadratic curve $(\mathrm{p}=0.022)$, with laying rate first increasing and then decreasing with increased SGYC supplementation. In addition, the laying rate in hens given $10 \mathrm{~g} / \mathrm{kg}$ supplements (85.1\%) was significantly higher than the control group (74.3\%) throughout the entire experiment period $(\mathrm{p}<0.05)$. Throughout both halves of the experimental period, increasing supplementation of SGYCs also led to first decreases and then increases in the feed conversion ratio, their relationship following a quadratic curve ( $\mathrm{p}=$ 0.007). Moreover, SGYC supplementation had significant effects on the feed conversion ratio $(\mathrm{p}<0.05)$. Specifically, the $10 \mathrm{~g} / \mathrm{kg}$ supplemental group had distinctly lower feed conversion ratio compared to the control. This result may be linked to the increase in egg weight: on average, eggs from the $10 \mathrm{~g} / \mathrm{kg}$ supplemental group weighed $0.23 \mathrm{~g}$ more compared to the control group. These results indicate that the addition of SGYCs to feeds for laying hens can effectively increase the frequency of egg laying and lower feed conversion ratio, and that such effects manifest in a gradual manner. Moreover, our results suggest that optimal effects are obtained when supplements are added at $10 \mathrm{~g} / \mathrm{kg}$ of feed. These results corroborate with previous studies such as Zhang et al [18], who used corn germ meal and wheat bran as substrates, fermented them into YC with $S$. cerevisiae, and found that increased egg-laying rate and reduced feed conversion ratio when a $0.3 \%$ supplementation of S. cerevisiae cultures was added to feeds.

Although SGYC supplementation did not significantly change the properties of the eggshell including shell strength, shell thickness, and shape index, it had a significant effect on the yolk color. Analysis of eggs collected on the 42nd day of the experimental period showed that yolks of eggs laid by hens from the treatment groups were significantly different in color compared to the control $(\mathrm{p}<0.01)$, and the RCF values of yolk color obtained from the $40 \mathrm{~g} / \mathrm{kg}$ group were as high as 8.90. The color of egg yolks is known to be a key factor in the marketing and sales of eggs, and golden or orange yolks with RCF $>8.0$ are typically more preferred by consumers [19]. Research has shown that most fat-soluble pigments can affect the color of egg yolks. Pigments responsible for the yellow color are typically carotenes [20], and most commonly xanthophylls $\left(\mathrm{C}_{40} \mathrm{H}_{56} \mathrm{O}_{2}\right)$. Common pigments present in feeds include lutein, zeaxanthin, and cryptoxanthin [21]. The enhanced yellow color in egg yolks from this study could be attributed to curcumin $\left(\mathrm{C}_{21} \mathrm{H}_{20} \mathrm{O}_{6}\right)$ found in ginger rhizomes. Curcumin is an isomer of lutein with very similar properties. Park et al [22] have also reported that the addition of $0.5 \%$ curcumin resulted in egg yolks with enhanced color.

\section{Apparent digestibility of nutrients}

The apparent digestibility of nutrients in laying hens are as shown in Table 3. Crude protein, crude fat, crude fiber, and ash all increased first and then decreased with increasing supplementation of SGYCs. Among them, the addition of SGYCs had a significant effect on the apparent digestibility of crude protein and crude fats in the feed $(\mathrm{p}<0.01)$, and the 
Table 2. Effects of SGYCs on production performance ${ }^{1)}$ and egg quality²) in laying hens

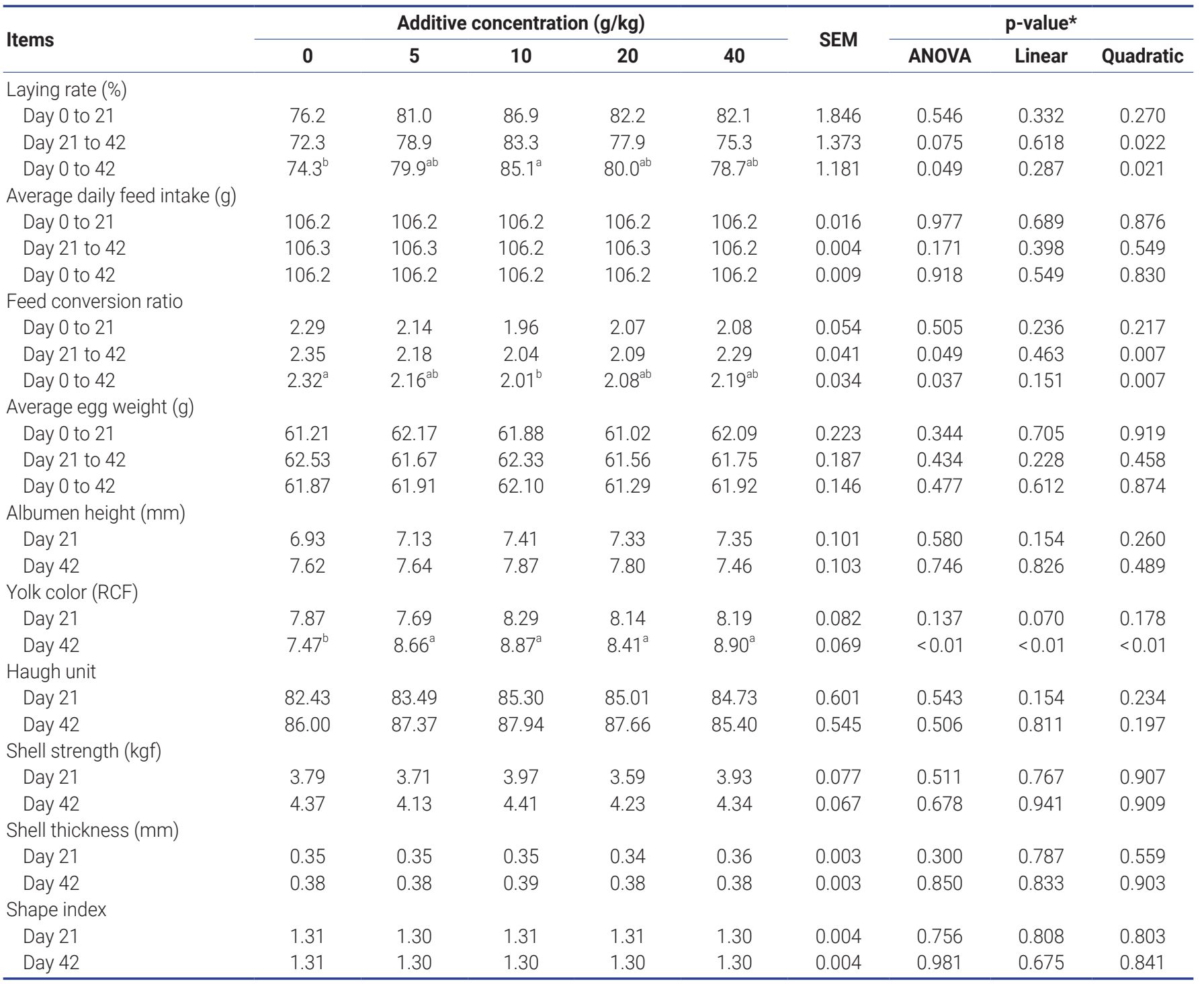

SGYCs, spent ginger yeast cultures; SEM, standard error of the means; ANOVA, analysis of variance.

1) Data are means for 4 replicates of 4 laying hens/replicate.

2) Data are means for 4 replicates of 3 eggs/replicate.

a,b The means within a row with different lowercase letters are significantly different $(p<0.05)$

* Responses (linear or quadratic) to different levels of SGYCs in the diet of laying hens.

$10 \mathrm{~g} / \mathrm{kg}$ supplement group had the highest apparent digestibility for all nutrients. This can be explained by several known properties of ginger. Active ingredients in ginger have been shown to antagonize muscarinic and histaminergic recep-

Table 3. Effects of SGYCs on apparent digestibility of nutrients in laying hens

\begin{tabular}{|c|c|c|c|c|c|c|c|c|c|}
\hline \multirow{2}{*}{ Items } & \multicolumn{5}{|c|}{ Additive concentration ( $\mathrm{g} / \mathrm{kg}$ ) } & \multirow{2}{*}{ SEM } & \multicolumn{3}{|c|}{ p-value* } \\
\hline & 0 & 5 & 10 & 20 & 40 & & ANOVA & Linear & Quadratic \\
\hline Crude fat (\%) & $83.92^{\mathrm{ab}}$ & $85.81^{a}$ & $87.68^{\mathrm{a}}$ & $84.48^{\mathrm{ab}}$ & $80.08^{b}$ & 0.740 & 0.007 & 0.109 & $<0.01$ \\
\hline Crude fiber $(\%)$ & 51.10 & 53.64 & 54.64 & 51.39 & 50.57 & 0.674 & 0.086 & 0.788 & 0.042 \\
\hline Crude protein (\%) & $58.37^{b}$ & $62.20^{\mathrm{ab}}$ & $63.42^{\mathrm{a}}$ & $60.98^{\mathrm{ab}}$ & $58.22^{b}$ & 0.675 & 0.009 & 0.755 & $<0.01$ \\
\hline Ash (\%) & 45.68 & 46.40 & 47.59 & 45.98 & 44.85 & 0.321 & 0.056 & 0.376 & 0.019 \\
\hline
\end{tabular}

SGYCs, spent ginger yeast cultures; SEM, standard error of the means; ANOVA, analysis of variance.

Data are means for 4 replicates of 2 laying hens/replicate.

${ }^{a, b}$ The means within a row with different lowercase letters are significantly different $(p<0.05)$

* Responses (linear or quadratic) to different levels of SGYCs in the diet of laying hens. 
tors and have a beneficial effect in facilitating gastrointestinal movement [23]. Studies have also reported that ginger can improve the activities of digestive enzymes such as lipase, sucrase and protease and thus facilitate digestion in the intestines [24]. As well, Zhang et al [18] demonstrated the effects of YCs in improving the digestion of laying hens by showing that adding $S$. cerevisiae cultures to the diet of laying hens can increase activities of digestive enzymes in the chyme while also decreasing the toxin levels in their plasma.

In addition, results of the present study show that during the latter half of the experimental period (day 21 to 42), the laying rate was significantly positively correlated with the apparent digestibility of crude fat, crude fiber, crude protein, and ash $(\mathrm{p}<0.05)$. Therefore, we conclude that the apparent digestibility of nutrients is closely related to the laying rate. Other studies have also shown that increasing the digestibility of nutrients in laying hens can improve rates of egg production [25].

\section{Routine blood indices and serum biochemical components}

Alterations in hematological indices including the decrease of RBC, HGB, and HCT are the main symptoms of anemia [26]. As shown in Table 4, the addition of SGYCs had no significant effect on blood indicators including RBC, MCV, and PLT ( $p>0.05)$. However, HGB and HCT levels followed a quadratic curve with increasing supplementation of SGYCs $(\mathrm{p}<0.05)$. This phenomenon indicates that SGYCs may have the potential to alleviate anemia, although requiring further research to elucidate. Results of this study also showed that there were no significant differences in the activities of ALT and AST in the serum between the groups ( $>>0.05)$. These two enzymes are excreted into the circulatory system by damaged liver cells, and thus their increased activity in the serum is a reliable indicator of liver damage or dysfunction [27]. The lack of differences in their activities in the present study suggests that the addition of SGYCs had no adverse effects on liver function.

As nutrients enter the blood after being absorbed and converted, the increased digestibility of nutrients in hens fed SGYCs is also reflected in nutrient levels in the serum. The results revealed that $\mathrm{TP}$ and $\mathrm{CHOL}$ content in the serum were significantly positively correlated with the apparent digestibility of proteins and fats, respectively $(p<0.01)$. Higher protein content in the serum is in turn correlated with egg albumen height $(\mathrm{p}<0.05)$. As albumen height is a main indicator in evaluating egg quality that reflects both nutrient levels and egg white consistency, the results also corroborate previous research that serum protein content is a key factor in determining egg quality [28].

\section{Serum antioxidant capacity}

As a byproduct of their metabolism, laying hens produce superoxide anions $\left(\mathrm{O}_{2}{ }^{--}\right)$, which are cytotoxic free radicals that can attack the polyunsaturated fatty acids in the biofilm, causing lipid oxidations and generating MDA [29]. MDA will then cause the cross-linking between membrane lipids and membrane proteins and ultimately disturb normal cell metabolism. SOD, GSH-Px, and CAT are the main endogenous antioxidant enzymes in animal cells and work in coordination to eliminate free radicals such as $\mathrm{O}_{2}{ }^{--}$[30]. Thus, the concentration of these molecules are indicators of cellular health and function.

As seen in Figure 1, with increased supplementation of SGYCs, activities of SOD, GSH-Px, and CAT all increased, and correspondingly levels of MDA decreased. Hens given $20 \mathrm{~g} / \mathrm{kg}$ supplementation had the highest enzymatic activities and all were significantly different compared to the control

Table 4. Effects of SGYCs on blood routine indices and serum biochemical parameters in laying hens

\begin{tabular}{|c|c|c|c|c|c|c|c|c|c|}
\hline \multirow{2}{*}{ Items } & \multicolumn{5}{|c|}{ Additive concentration ( $\mathrm{g} / \mathrm{kg})$} & \multirow{2}{*}{ SEM } & \multicolumn{3}{|c|}{ p-value* } \\
\hline & 0 & 5 & 10 & 20 & 40 & & ANOVA & Linear & Quadratic \\
\hline $\operatorname{RBC}\left(\times 10^{6} \mu \mathrm{L}\right)$ & 2.50 & 2.59 & 2.60 & 2.70 & 2.59 & 0.028 & 0.322 & 0.172 & 0.179 \\
\hline $\mathrm{HGB}(\mathrm{g} / \mathrm{dL})$ & 13.76 & 14.48 & 14.60 & 15.15 & 14.48 & 0.159 & 0.111 & 0.067 & 0.039 \\
\hline HCT (\%) & 29.48 & 30.40 & 31.30 & 31.73 & 26.69 & 0.316 & 0.093 & 0.437 & 0.035 \\
\hline $\operatorname{MCV}(f L)$ & 115.40 & 116.90 & 117.50 & 117.80 & 116.00 & 0.566 & 0.647 & 0.602 & 0.301 \\
\hline $\operatorname{PLT}\left(\times 10^{3} \mu \mathrm{L}\right)$ & 14.11 & 14.00 & 14.44 & 14.56 & 14.00 & 0.756 & 0.999 & 0.953 & 0.982 \\
\hline GLU (mmol/L) & 12.09 & 11.31 & 11.53 & 11.50 & 11.44 & 0.236 & 0.879 & 0.515 & 0.681 \\
\hline $\operatorname{ALT}(\mathrm{U} / \mathrm{L})$ & 1.1 & 1.1 & 1.0 & 1.1 & 1.1 & 0.137 & 0.997 & 1.000 & 0.970 \\
\hline AST (U/L) & 196.43 & 194.57 & 187.14 & 203.29 & 206.00 & 3.137 & 0.361 & 0.218 & 0.219 \\
\hline $\mathrm{TP}(\mathrm{g} / \mathrm{L})$ & 52.37 & 53.89 & 54.49 & 52.83 & 49.13 & 0.827 & 0.314 & 0.174 & 0.089 \\
\hline $\mathrm{CHOL}(\mathrm{mmol} / \mathrm{L})$ & 2.33 & 2.70 & 2.79 & 2.34 & 2.32 & 0.096 & 0.332 & 0.581 & 0.215 \\
\hline
\end{tabular}

SGYCs, spent ginger yeast cultures; SEM, standard error of the means; ANOVA, analysis of variance; RBC, red blood cells; HGB, hemoglobin; HCT, hematocrit; MCV, mean corpuscular volume; PLT, platelet count; GLU, glucose; ALT, alanine aminotransferase; AST, aspartic acid aminotransferase; TP, total protein; $\mathrm{CHOL}$, total cholesterol.

Data are means for 4 replicates of 3 laying hens/replicate.

* Responses (linear or quadratic) to different levels of SGYCs in the diet of laying hens. 


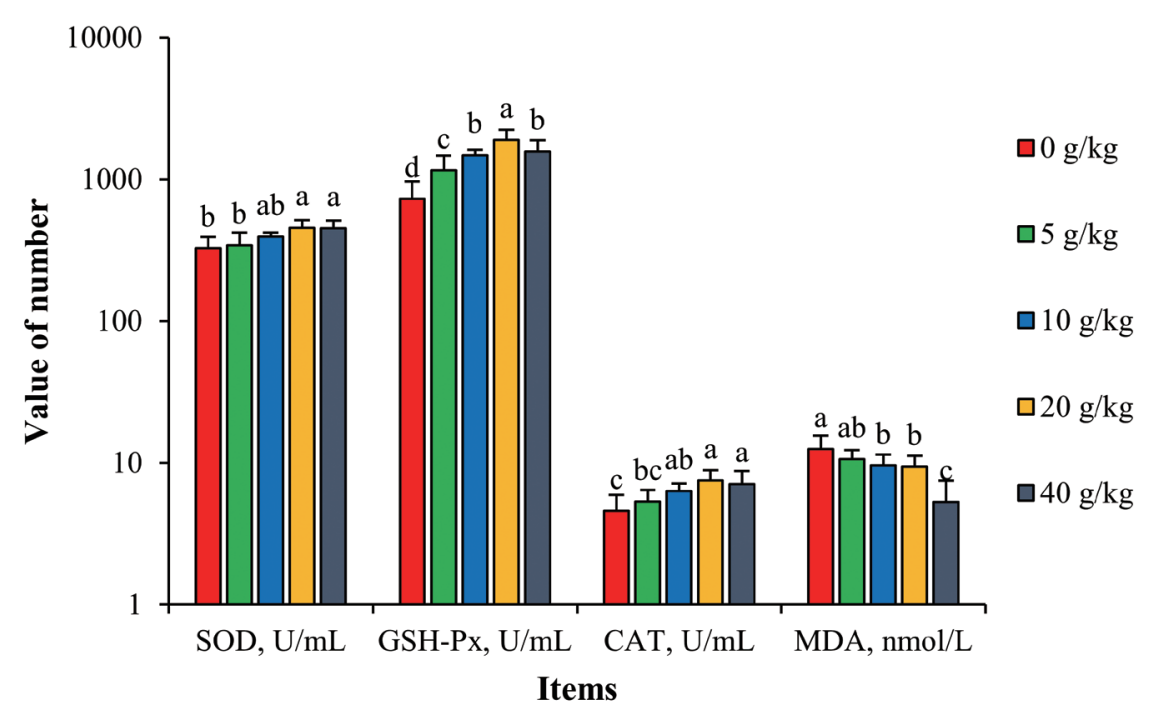

Figure 1. Effects of SGYCs on serum antioxidant capacity in laying hens. SGYCs, spent ginger yeast cultures; SOD, superoxide dismutase; GSHPx, glutathione peroxidase; CAT, peroxidase; MDA, malondialdehyde. Values are presented as means \pm standard error. ${ }^{\text {a-d }}$ Columns with different lowercase letters differ significantly $(p<0.05)$.

$(\mathrm{p}<0.05)$. On the other hand, the treatment group given 40 $\mathrm{g} / \mathrm{kg}$ supplementation had the lowest levels of MDA, up to $28.4 \%$ lower than the control $(p<0.05)$. These results show that SGYCs can facilitate anti-oxidizing reactions in hens, lowering levels of MDA and thus reducing physiological abnormalities. Similar observations have been made by Zhao et al [31], who added powdered ginger to the feed of laying hens. These effects can be attributed to the various antioxidants found in ginger, such as [6]-gingerol, which inhibits lipid oxidation [32], or flavonoids, which can remove free radicals such as hydroxyls and superoxide anions.

\section{Intestinal microbiota}

The intestinal microbiota community structure is shown in Figure 2, the dominant phyla of gut bacterial were Firmicutes, Bacteroidetes, and Proteobacteria in all treatment groups, which are the same groups reported to be most dominant in the intestinal tract of healthy poultry [33]. Thus, it appears that the addition of SGYCs did not adversely affect the intestinal microbiota of laying hens. Firmicutes was the most dominant phylum in all treatment groups and are known for degrading carbohydrates in the intestine. Considering that carbohydrates are the main source of energy for all organisms, it is unsurprising that Firmicutes have a great advantage. These observations are also consistent with previous reports such as those of Shang et al [27]. Additionally, studies have reported that there are more protein-degrading bacteria species in Bacteroidetes [34]. In this study, the relative abundance of Bacteroidetes was higher in the $10 \mathrm{~g} / \mathrm{kg}$ supplement group when compared with the control group (19.01\% vs $11.68 \%)$, which may explain the higher apparent digestibility of proteins in the groups given $10 \mathrm{~g} / \mathrm{kg}$ supplements, although these changes were not significant ( $p>0.05)$. It has also been reported that ingredients including polyphenols, flavonoids, and dietary fiber changed the relative abundance of Firmicutes and Bacteroidetes in the intestine [35,36], and SGYCs are rich in these ingredients. Additionally, the ratio of Firmicutes to Bacteroidetes is known to be negatively correlated with fat metabolism [37]. The results of the present study also follow this pattern: the $10 \mathrm{~g} / \mathrm{kg}$ supplement group had the lowest Firmicutes to Bacteroidetes ratio (4.04) and the highest apparent fat digestibility $(87.68 \%)$, which is followed by the 5 $\mathrm{g} / \mathrm{kg}$ supplement group with Firmicutes to Bacteroidetes ratio of 4.06 and fat apparent digestibility of $85.81 \%$. The above results are also concurrent with Liu et al, who found that the

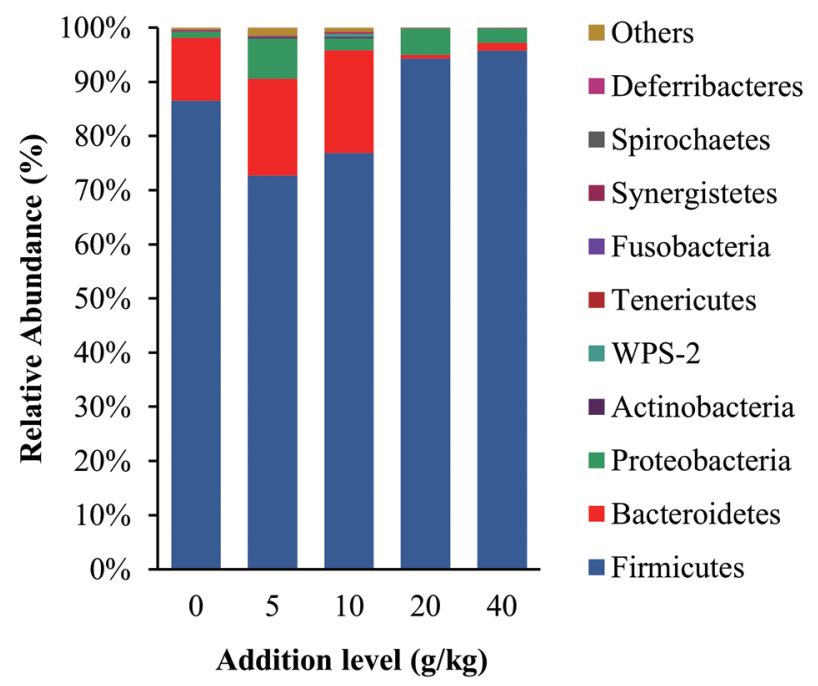

Figure 2. Compositional changes in the intestinal microbiota of laying hens at the phylum levels. 
improvement of various production aspects in laying hens given commercial YCs, including the quality of eggs, immune function, antioxidant capacity, reproduction efficiency, digestion, and absorption capacity, were linked to the change in the relative abundance of gut microbiota [38].

\section{CONCLUSION}

The current evidence shows that dietary supplementation of SGYCs to the feed of laying hens can improve laying rates and decrease feed conversion ratio, and such effects are the most prominent when they are added at $10 \mathrm{~g} / \mathrm{kg}$ of feed. Eggs laid by treatment group hens also had yolks with enhanced color. Additionally, enzymatic activity of antioxidants in the serum also significantly increased, suggesting a lower occurrence of physiological abnormalities. These findings all support the potential application of SGYCs as a feed supplement that benefits laying hens. The potential mechanisms through which SGYCs influence production performance and egg quality of laying hens are summarized in Supplementary Figure S1: the addition of SGYCs firstly changes the relative abundance of dominant microbiota in the intestines of laying hens. This improves the apparent digestibility of nutrients in the feed, which promotes egg laying and increases the content of nutrients in the blood, especially the TP content. The increased protein content in the serum also improves protein quality of the eggs.

\section{CONFLICT OF INTEREST}

We certify that there is no conflict of interest with any financial organization regarding the material discussed in the manuscript.

\section{FUNDING}

The authors are grateful for the support by the key research and development program of Shandong Province (2017GNC 10105).

\section{SUPPLEMENTARY MATERIAL}

Supplementary file is available from: https://doi.org/10.5713/ ab.21.0514

Supplementary Figure S1. Potential mechanism of SGYCs affecting egg production and egg quality in laying hens.

\section{REFERENCES}

1. Anton M. Egg yolk: structures, functionalities and processes. J Sci Food Agric 2013;93:2871-80. https://doi.org/10.1002/ jsfa.6247

2. Zhao Y, Feng F, Yang Y, Xiong C, Xu M, Tu Y. Gelation behavior of egg yolk under physical and chemical induction: a review. Food Chem 2021;355:129569. https://doi.org/10. 1016/j.foodchem.2021.129569

3. Gautron J, Dombre C, Nau F, Feidt C, Guillier L. Review: Production factors affecting the quality of chicken table eggs and egg products in Europe. Animal 2022;16:100425. https://doi.org/10.1016/j.animal.2021.100425

4. Liu YZ, Chen X, Zhao W, et al. Effects of yeast culture supplementation and the ratio of non-structural carbohydrate to fat on rumen fermentation parameters and bacterial-community composition in sheep. Anim Feed Sci Technol 2019;249:6275. https://doi.org/10.1016/j.anifeedsci.2019.02.003

5. Gómez S, Angeles ML, Mojica MC, Jalukar S. Combination of an enzymatically hydrolyzed yeast and yeast culture with a direct-fed microbial in the feeds of broiler chickens. AsianAustralas J Anim Sci 2012;25:665-73. https:/doi.org/10.5713/ ajas.2011.11316

6. Ma YB, Zhou JM, Zhang HJ, et al. Effects of dietary yeast culture of white distiller's grains in different energy level diets on growth performance, carcass composition and serum biochemical indices of broilers. Chin J Anim Nutr 2018;30: 274-83. https://doi.org/10.3969/j.issn.1006-267x.2018.01.033

7. Yalçin S, Özsoy B, Erol H, Yalçin S. Yeast culture supplementation to laying hen diets containing soybean meal or sunflower seed meal and its effect on performance, egg quality traits, and blood chemistry. J Appl Poult Res 2008;17:22936. https://doi.org/10.3382/japr.2007-00064

8. Kiyama R. Nutritional implications of ginger: chemistry, biological activities and signaling pathways. J Nutr Biochem 2020;86:108486. https://doi.org/10.1016/j.jnutbio.2020.108486

9. Jafarzadeh A, Nemati M. Therapeutic potentials of ginger for treatment of multiple sclerosis: a review with emphasis on its immunomodulatory, anti-inflammatory and antioxidative properties. J Neuroimmunol 2018;324:54-75. https:// doi.org/10.1016/j.jneuroim.2018.09.003

10. Wen HS, Yan PC, Zhang JH, Chen ZY, Chung HY. Antioxidant activities of ginger extract and its constituents toward lipids. Food Chem 2018;239:1117-25. https://doi.org/10.1016/j. foodchem.2017.07.055

11.Zhang BB, Dai YA, Liao ZX, Ding LS. Three new antibacterial active diarylheptanoids from Alpinia officinarum. Fitoterapia 2010;81:948-52. https://doi.org/10.1016/j.fitote.2010.06.015

12.Huang FY, Deng T, Meng LX, Ma XL. Dietary ginger as a traditional therapy for blood sugar control in patients with type 2 diabetes mellitus: a systematic review and metaanalysis. Medicine 2019;98:e15054. https://doi.org/10.1097/ MD.0000000000015054

13. Hsiang CY, Lo HY, Huang HC, Li CC, Wu SL, Ho TY. Ginger extract and zingerone ameliorated trinitrobenzene sulphonic acid-induced colitis in mice via modulation of nuclear factor- 
$\kappa b$ activity and interleukin $-1 \beta$ signalling pathway. Food Chem 2013;136:170-7. https://doi.org/10.1016/j.foodchem. 2012.07.124

14. National Reasearch Council. Nutrient requirements of poultry. 9th ed. Washington, DC, USA: National Academy Press; 1994.

15.Liu ZL, Xue JJ, Huang XF, et al. Effect of feeding frequency on the growth performance, carcass traits, and apparent nutrient digestibility in geese. Poult Sci 2020;99:4818-23. https://doi.org/10.1016/j.psj.2020.06.024

16. AOAC International. Official methods of analysis of AOAC International. 18th ed. Arlington, VA, USA: AOAC International; 2005.

17. Callahan BJ, Mcmurdie PJ, Rosen MJ, Han AW, Johnson AJ, Holmes SP. Dada2: high-resolution sample inference from illumina amplicon data. Nat Methods 2016;13:581-3. https:// doi.org/10.1038/nmeth.3869

18.Zhang JC, Chen P, Zhang C, et al. Yeast culture promotes the production of aged laying hens by improving intestinal digestive enzyme activities and the intestinal health status. Poult Sci 2020;99:2026-32. https://doi.org/10.1016/j.psj.2019. 11.017

19.Lokaewmanee K, Yamauchi K, Komori T, Saito K. Enhancement of egg yolk color by paprika combined with a probiotic. J Appl Poult Res 2011;20:90-4. https://doi.org/10.3382/japr. 2009-00140

20. Galobart J, Sala R, Rincon-Carruyo X, Manzanilla EG, Vila B, Gasa J. Egg yolk color as affected by saponification of different natural pigmenting sources. J Appl Poult Res 2004;13:32834. https://doi.org/10.1093/japr/13.2.328

21.Surai PF, Speake BK, Sparks NHC. Carotenoids in avian nutrition and embryonic development. 1. absorption, availability and levels in plasma and egg yolk. J Poult Sci 2001; 38:1-27. https://doi.org/10.2141/jpsa.38.1

22.Park SS, Kim JM, Kim EJ, Kim HS, An BK, Kang CW. Effects of dietary turmeric powder on laying performance and egg qualities in laying hens. Poult Sci 2012;39:27-32. https://doi. org/10.5536/KJPS.2012.39.1.027

23. Walstab J, Krüger D, Stark T, et al. Ginger and its pungent constituents non-competitively inhibit activation of human recombinant and native 5-HT3 receptors of enteric neurons. Neurogastroenterol Motil 2013;25:439-e302. https://doi.org/ 10.1111/nmo.12107

24.Platel K, Srinivasan K. Influence of dietary spices and their active principles on pancreatic digestive enzymes in albino rats. Mol Nutr Food Res 2000;44:42-6. https://doi.org/10.1002/ (SICI)1521-3803(20000101)44:1<42::AID-FOOD42>3.0.CO; 2-D

25.Ghasemian M, Jahanian R. Dietary mannan-oligosaccharides supplementation could affect performance, immunocompetence, serum lipid metabolites, intestinal bacterial populations, and ileal nutrient digestibility in aged laying hens. Anim
Feed Sci Technol 2016;213:81-9. https://doi.org/10.1016/j. anifeedsci.2015.12.012

26. Pan C, Yan MH, Jin H, Guo HQ, Han XD. Chronic exposure to MC-LR increases the risks of microcytic anemia: Evidence from human and mice. Environ Pollut 2021;288:117966. https:// doi.org/10.1016/j.envpol.2021.117

27.Shang HM, Zhao JC, Dong XQ, et al. Inulin improves the egg production performance and affects the cecum microbiota of laying hens. Int J Biol Macromol 2020;155:1599-609. https:// doi.org/10.1016/j.ijbiomac.2019.11.137

28. Elkin RG, Freed M, Watkins BA, Srebnik M, Kieft KA, Newton RS. Evaluation of two novel biochemicals on plasma and egg yolk lipid composition and laying hen performance. Poult Sci 1993;72:513-20. https://doi.org/10.3382/ps.0720513

29. Dimitrios T. Assessment of lipid peroxidation by measuring malondialdehyde (MDA) and relatives in biological samples: analytical and biological challenges. Anal Biochem 2017;524: 13-30. https://doi.org/10.1016/j.ab.2016.10.021

30.Ibtisham F, Nawab A, Niu YF, et al. The effect of ginger powder and chinese herbal medicine on production performance, serum metabolites and antioxidant status of laying hens under heat-stress condition. J Therm Biol 2019;81:20-4. https:// doi.org/10.1016/j.jtherbio.2019.02.002

31.Zhao X, Yang ZB, Yang WR, Wang Y, Jiang SZ, Zhang GG. Effects of ginger root (zingiber officinale) on laying performance and antioxidant status of laying hens and on dietary oxidation stability. Poult Sci 2011;90:1720-7. https://doi.org/ 10.3382/ps.2010-01280

32.Ippoushi K, Azuma K, Ito H, Horie H, Higashio H. 6-Gingerol inhibits nitric oxide synthesis in activated J774.1 mouse macrophages and prevents peroxynitrite-induced oxidation and nitration reactions. Life Sci 2003;73:3427-37. https:// doi.org/10.1016/j.lfs.2003.06.022

33. Best AA, Porter AL, Fraley SM, Fraley GS. Characterization of gut microbiome dynamics in developing pekin ducks and impact of management system. Front Microbiol 2017;7: 2125. https://doi.org/10.3389/fmicb.2016.02125

34.Panasevich MR, Meers GM, Linden MA, et al. High-fat, high-fructose, high-cholesterol feeding causes severe nash and cecal microbiota dysbiosis in juvenile ossabaw swine. Am J Physiol Endocrinol Metab 2018;314:E78-E92. https:// doi.org/10.1152/ajpendo.00015.2017

35.Li S, You JM, Wang ZR, et al. Curcumin alleviates high-fat diet-induced hepatic steatosis and obesity in association with modulation of gut microbiota in mice. Food Res Int 2021;143:110270. https://doi.org/10.1016/j.foodres.2021. 110270

36.Xu RY, Liu Y, Wang J, Liu JJ, Su Y, Zhu WY. Effects of the different dietary fibers on luminal microbiota composition and mucosal gene expression in pig colons. J Funct Foods 2019;59:71-9. https://doi.org/10.1016/j.jff.2019.05.035

37. Guo X, Xia X, Tang R, Zhou J, Zhao H, Wang K. Development 
of a real-time PCR method for firmicutes and bacteroidetes in faeces and its application to quantify intestinal population of obese and lean pigs. Lett Appl Microbiol 2008;47:367-73. https://doi.org/10.1111/j.1472-765X.2008.02408.x
38.Liu YC, Cheng X, Zhen WR, et al. Yeast culture improves egg quality and reproductive performance of aged breeder layers by regulating gut microbes. Front Microbiol 2021;12: 633276. https://doi.org/10.3389/fmicb.2021.633276 